\title{
Reference-free digital shadowgraphy using a moving BOS background
}

\author{
A. D. Gardner ${ }^{1}$ - M. Raffel ${ }^{1}$. C. Schwarz ${ }^{1}$. J. N. Braukmann ${ }^{1}$ - C. C. Wolf ${ }^{1}$
}

Received: 20 November 2019 / Revised: 7 December 2019 / Accepted: 12 December 2019 / Published online: 21 January 2020

(c) The Author(s) 2020

\begin{abstract}
A method of measuring density second derivative (shadowgraph) as a variant of background oriented schlieren (BOS) is presented. Pairs of images of a moving background with a small time difference are analysed using moving-window crosscorrelation, giving the second derivative of density in the axis of background travel. The advantage over standard BOS is that no reference image is required. In contrast to the existing "reference-free BOS" method, no double-image is produced, making the data interpretation simpler. The sum-of-correlation (ensemble correlation) method can be used on an image series, where the flow is constant over the series time, to significantly reduce the size of the cross-correlation window. The reduction in the window size and sharpening of the resulting image is similar to that achieved by optical flow, but this method is easily automatable, without knowledge of the flow, and can be used with any existing BOS analysis software.
\end{abstract}

\section{Graphic abstract}
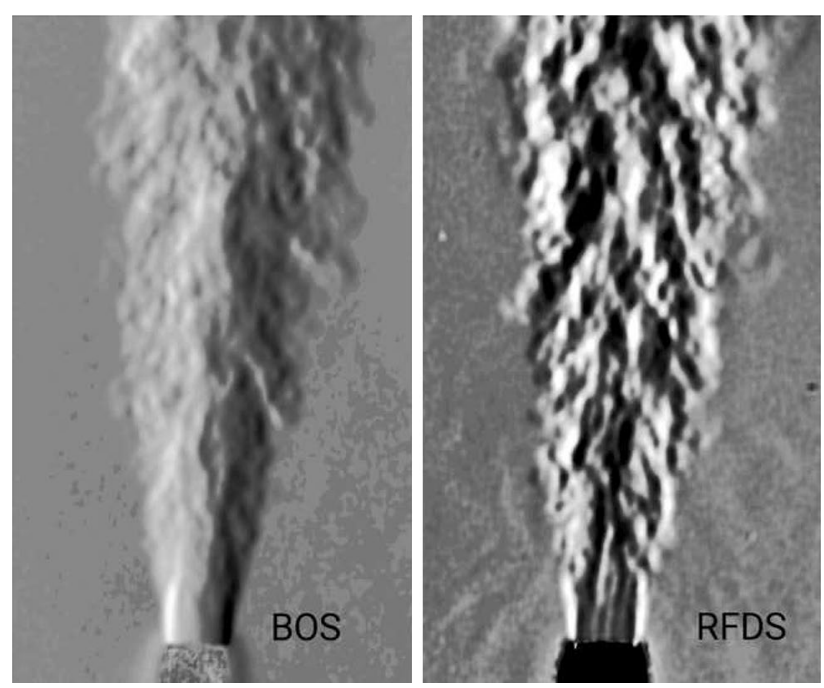

\section{List of symbols}

$f \quad$ Camera focal length $(=200 \mathrm{~mm})$

$F \# \quad$ Camera f-stop $(\mathrm{F} \#=22)$

$n, n_{0} \quad$ Object, background refractive index

$\rho \quad$ Density $\left(\mathrm{kg} / \mathrm{m}^{3}\right)$

$x \quad$ Coordinate in breadth, movement axis

\section{A. D. Gardner}

Tony.Gardner@dlr.de

1 German Aerospace Center (DLR), Institute of Aerodynamics and Flow Technology, Bunsenstrasse 10, 37073 Göttingen, Germany y Coordinate vertically

$z \quad$ Coordinate in depth between camera and background

$z_{A} \quad$ Camera-object distance $\left(z_{A}=1440 \mathrm{~mm}\right)$

$z_{D} \quad$ Object-background distance $\left(z_{D}=880 \mathrm{~mm}\right)$

\section{Introduction}

Background oriented schlieren (BOS) is a common density gradient imaging technique, with a large number of variants, summarised in Raffel (2015). A camera is focused on a 
background with a low self-correlating pattern ("random dot pattern"), which is visualised once without flow and once with flow. A moving-window cross-correlation algorithm, identical to that used for particle image velocimetry (PIV) is used to compare the two images, and the movement of the background is related to the refractive index by:

$\Delta y=f\left(\frac{z_{D}}{z_{D}+z_{A}-f}\right) \frac{1}{n_{0}} \int_{0}^{l} \frac{\partial n}{\partial y} \mathrm{~d} z$,

and an equivalent equation gives the movement in the $\mathrm{x}$ axis. The background movement $\Delta y$ is a function of the $\mathrm{z}$ dimensions as detailed in Fig. 1, the camera focal length $f$ and the index of refraction of the undisturbed background $n_{0}$ and of the object $n$, which are related to the density via the Gladstone-Dale equation. The refractive index is integrated over the line of sight, and the background movement is a function of the first derivative of the density, with the $x$ and $y$ components separable in the image plane. The second derivative can be acquired by differentiating the data a second time during post-processing, see Settles (2018), and this method is often used for vortex visualisation by BOS to improve the image contrast, see Bauknecht et al. (2016).

In contrast to classical schlieren techniques, the BOS technique has several drawbacks, as detailed by Settles and Hargather (2017):

1. The evaluation window size limits the minimum size of object which can be visualised by a given setup, imposing a spatial filter on the data.

2. The requirement for a reference image with undisturbed flow can be difficult to realize for some test cases.

3. The requirement to focus on the background leads to an irreducible unsharpness of the imaged object, imposing an additional spatial filter on the data.

For point 1 the size of the evaluation window needed is a function of the signal to noise level of the images, but also generally several background dots will be required to get a good cross-correlation peak. One approach to reducing this

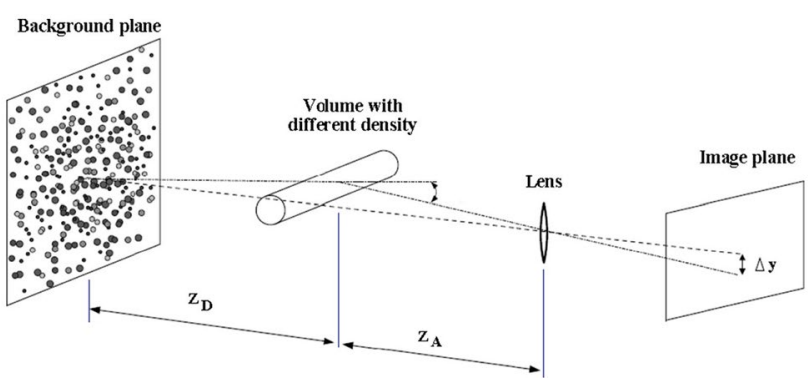

Fig. 1 BOS Arrangement and lengths, modified from Raffel (2015) requirement for static flows is to use optical flow algorithms for the analysis of a series of BOS images, as detailed by Hill and Haering (2017) for an aircraft flying in front of the sun, and by Smith et al. (2017) for BOS from a follower aircraft. As noted in those papers, a problem with optical flow algorithms as currently implemented is a lack of robustness compared with window cross-correlation.

For point 2 it has been noted that a reference image can be difficult to realize for outdoor measurements, when the natural light changes between the reference and measurement images leads to problems with the cross-correlation, see Bauknecht et al. (2014). Additionally, when shooting from a follower aircraft, no simple reference image is available, since the background is moving, see Bauknecht et al. (2016). The solution used in these cases is a "Reference free BOS", in which two images are taken with a separation which is long compared to the movement, so that the disturbances of the second image have moved onto undisturbed background in the first image and vice-versa, creating a double-image.

\section{Reference-free digital shadowgraphy}

Reference-free digital shadowgraphy (RFDS) uses essentially the same setup as Fig. 1, with the exception that the background is moved. A pair of images is taken with a small time difference, so that the flow is steady on the time frame of the image pair, in the same logic as is used for PIV. As seen in Fig. 2, if the position of an object on the background moves from position $x_{1}$ to $x_{2}$ then between the two images it will be seen through a different part the density object. Subtracting the two images will then give the second derivative at the position midway between the two measurement positions. The movement of the background should be a small fraction of the

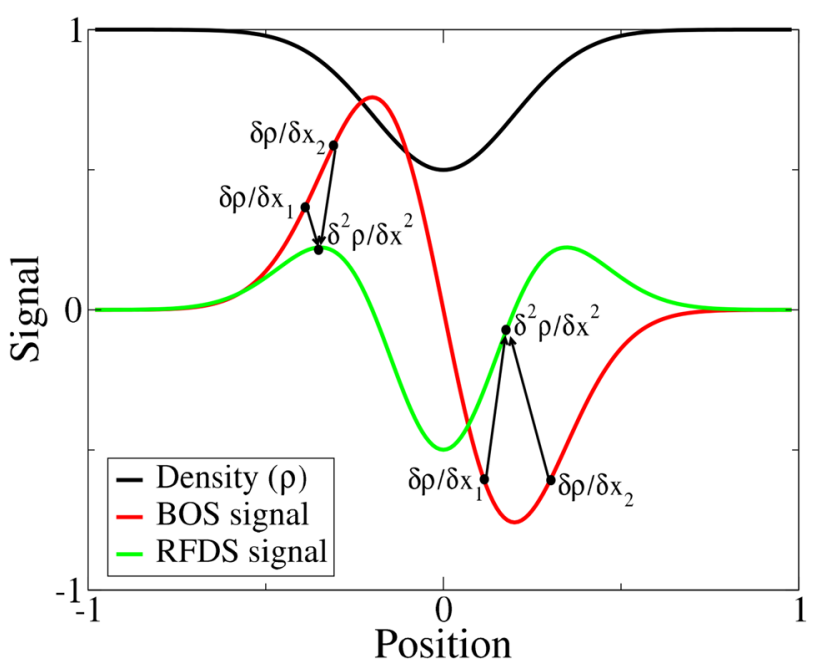

Fig. 2 Reference-free digital shadowgraphy idea 
object size between images 1 and 2, so that for a background movement in the $x$-axis Eq. 1 becomes:

$\Delta x=f\left(\frac{z_{D}}{z_{D}+z_{A}-f}\right) \frac{1}{n_{0}} \int_{0}^{l}\left[\frac{\partial n_{2}}{\partial x_{2}}-\frac{\partial n_{1}}{\partial x_{1}}\right] \mathrm{d} z$,

or when $x_{2}-x_{1} \rightarrow 0$ :

RFDS Signal $=\frac{\Delta x}{x_{2}-x_{1}}-1 \propto \int_{0}^{l}\left[\frac{\partial^{2} \rho}{\partial x^{2}}\right] \mathrm{d} z$.

This is similar to the relationship for standard shadowgraphy, but the second derivative is only found in the axis of the background movement, rather than as an addition of both axes. Normalisation by the background movement velocity is necessary to prevent a dependence of the signal sign on the background movement direction, and 1 is subtracted to have the signal around zero as is normal for shadowgraph images.

Figure 2 shows a qualitative illustration of the expected BOS signal $(\alpha \partial \rho / \partial x)$ as the red line, produced over an object with varying density $(\rho)$ in the negative direction (as expected from a vortex or heated jet), as shown by the black line. If the data from two locations in the BOS measurement are subtracted, an RFDS signal is produced $\left(\propto \partial^{2} \rho / \partial x^{2}\right)$ as the green line. The strength of the background velocity signal increases with increasing background movement between images. The spatial resolution (or spatial filter) is limited by the greatest of three lengths: The unsharpness at the measurement plane, the background movement between two images and the crosscorrelation window size. Ideally all three should be chosen to be around the same size.

Additionally if a series of images is taken during the time that the flow can be considered steady, either by using fast acquisition, or a steady flow, then the ensemble correlation of Meinhart et al. (2000) can be used to reduce the window size to the range $4 \times 4-8 \times 8$ pixels.

A similar subtraction method is visible in Settles and Hargather (2017), Fig. 3b, however the moving background method used here is new. Multiple images have been used previously for schlieren velocimetry by Mittelstaedt et al. (2010), but here the ensemble correlation was not used. The AIRBOS method of Smith et al. (2017) used optical flow algorithms to get similar results.

\section{Setup}

A Zeiss Milvus 2/100M ZF.2 lens with a focal length doubler was used and an f-stop of $F \#=22$ was used. This led to a resolution of $9.4 \mathrm{px} / \mathrm{mm}$ in the image, and an unsharpness measured at the object plane of $1.25 \mathrm{~mm}$ (12 px) in both $x$ and $y$ axes. A jet of heated air at $140{ }^{\circ} \mathrm{C}$ ejected from a

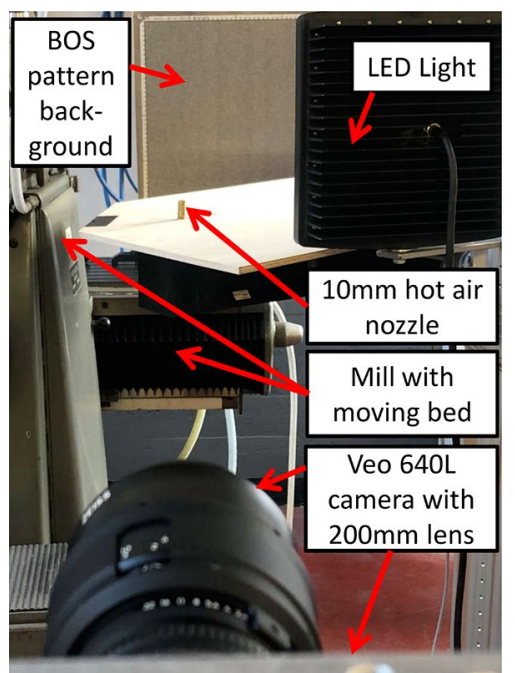

Fig. 3 Reference-free digital shadowgraphy setup

$10 \mathrm{~mm} / 14 \mathrm{~mm}$ ID/OD nozzle was used as the density object, meaning that there were 100-200 pixels over the density object, depending on the distance from the nozzle, and the unsharpness was around $5-10 \%$ of the density object size. In this test case the background was moved using the bed movement of a mill, as seen in Fig. 3, but a rotating disk background could also be envisaged. The linear movement rate was $20 \mathrm{~mm} / \mathrm{s}$, or $8 \mathrm{px}$ between images, less than the image unsharpness. The values for the setup are summarised in Table 1.

The data was analysed using DaVis 10.1 from LaVision (2019). A multipass algorithm was used with square crosscorrelation windows starting at $64 \times 64$ and reducing in size to the final value. No additional smoothing or data improvement was used.

Table 1 Experiment setup

\begin{tabular}{ll}
\hline Value & Meaning \\
\hline 22 & F\# for depth of field purposes \\
$f=200 \mathrm{~mm}$ & Lens focal length \\
$24 \mathrm{~Hz}$ & Image frequency \\
$140^{\circ}$ & Temperature of heated air jet \\
Veo $640 \mathrm{~L}$ & Camera \\
$2560 \times 1600 \mathrm{px}$ & Camera resolution \\
$800 \mu \mathrm{s}$ & Camera framing time \\
$z_{A}=1440 \mathrm{~mm}$ & Camera-object distance \\
$z_{D}=880 \mathrm{~mm}$ & Object-background distance \\
$9.4 \mathrm{px} / \mathrm{mm}$ & Camera resolution \\
$1.25 \mathrm{~mm} / 12 \mathrm{px}$ & Minimum resolvable object due to unsharpness \\
$8 \mathrm{px}$ & Background movement between images \\
\hline
\end{tabular}




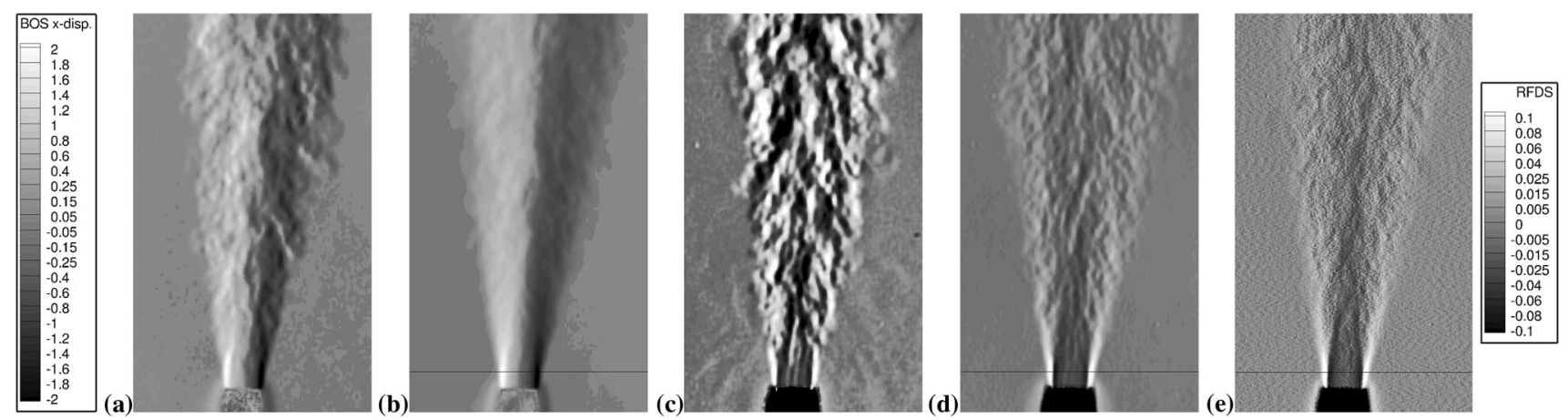

Fig. 4 BOS and RFDS image examples: a single image standard BOS using $16 \times 16$ px window and $75 \%$ overlap b standard BOS averaged over 20 images c single image RFDS using 16x16 px window and

\section{Results}

Figure 4 shows the results of the image analysis. The background movement in the $x$-direction for BOS images (equivalent to a vertical knife-edge schlieren) is shown for an instantaneous image in Fig. $4 \mathrm{a}$ and for an average of 20 images in Fig. 4b. The window size was $16 \times 16$ px, which was slightly larger than the image unsharpness. A single reference-free digital shadowgraph image is in Fig. 4c, from a pair of images $1 / 24 \mathrm{~s}$ apart. The fine structure of the turbulent jet is visible at the top of the image, and is comparable with the topology in Fig. 4a, although the image is taken at a different time point. In Fig. $4 d$ an average of 20 RFDS images is shown, and is comparable with the BOS result in Fig. 4b. The final result in Fig. 4e is for the ensemble correlation, and here the evaluation window was reduced to $6 \times 6$ with $75 \%$ overlap, which is definitely smaller than both the image unsharpness and the background movement. For this final image, the limit on the spatial resolution is given by the image unsharpness.

For the averaged data in Fig. 4, the data was extracted along the lines in the laminar jet region near to the jet exit. In this area the result is very similar between the averaged and the single-image data since the flow is relatively constant. The results of the cuts are in Fig. 5, and it can be seen that the expected result from Fig. 2 is generally reproduced, although the shape of the jet is clearly different to the nominal Gaussian form. It can be seen that for this particular flow that the window size of $16 \mathrm{px}$ adequately resolves the flow features, and the finer resolution of the ensemble correlation slightly changes the peak heights, but the result is otherwise qualitatively unchanged.
$75 \%$ overlap d RFDS averaged over 20 images e RFDS ensemble average over 20 images using $6 \times 6 \mathrm{px}$ window and $75 \%$ overlap

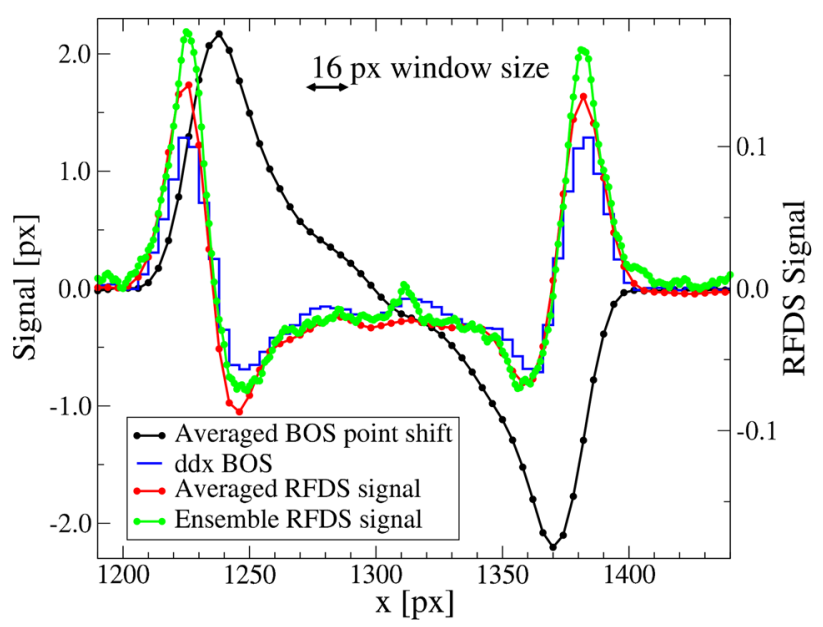

Fig. 5 Lines extracted from Fig. 4

\section{Conclusions}

A new method of measuring density second derivative (shadowgraph) as a variant of background oriented schlieren (BOS) was presented and shown to be practically measurable. the main results are:

1. No reference image is required for cases of linear background movement and appropriately-matching camera frame rate. Analysis of an image-pair acquired similarly to PIV is sufficient.

2. The analysis using moving cross-correlation windows is robust in existing software.

3. A reduction in spatial resolution can be avoided by keeping the background movement less than the optical unsharpness. 
4. Ensemble correlation can be used on image series for flows which are steady within the series time to significantly increase the measurement resolution.

Reference-free digital shadowgraphy (RFDS) is a potentially useful measurement method for some situations where traditional BOS is difficult to realise.

Acknowledgements Open Access funding provided by Projekt DEAL. This work was financed by the DLR project FAST-Rescue. The assistance of Markus Krebs is gratefully acknowledged.

Open Access This article is licensed under a Creative Commons Attribution 4.0 International License, which permits use, sharing, adaptation, distribution and reproduction in any medium or format, as long as you give appropriate credit to the original author(s) and the source, provide a link to the Creative Commons licence, and indicate if changes were made. The images or other third party material in this article are included in the article's Creative Commons licence, unless indicated otherwise in a credit line to the material. If material is not included in the article's Creative Commons licence and your intended use is not permitted by statutory regulation or exceeds the permitted use, you will need to obtain permission directly from the copyright holder. To view a copy of this licence, visit http://creativecommons.org/licenses/by/4.0/.

\section{References}

Bauknecht A, Merz CB, Raffel M (2016) Airborne visualization of helicopter blade tip vortices. J Vis. https://doi.org/10.1007/s1265 $0-016-0389-z$
Bauknecht A, Merz CB, Landolt A, Meier AH, Raffel M (2014) Bladetip vortex detection in maneuvering flight using the backgroundoriented Schlieren technique. J Aircraft. https://doi.org/10.2514/1. C032672

GmbH LaVision (2019) Product manual for DaVis 10.1: flow master D10.1. LaVision GmbH, Göttingen

Hill MA, Haering EA (2017) Ground-to-air flow visualization using solar calcium-K line background-oriented schlieren. Exp Fluids. https://doi.org/10.1007/s00348-016-2285-7

Meinhart CD, Wereley ST, Santiago JG (2000) A PIV algorithm for estimating time-averaged velocity fields. J Fluids Eng. https://doi. org/10.1115/1.483256

Mittelstaedt E, Davaille A, van Keken PE, Gracias N, Escartin J (2010) A noninvasive method for measuring the velocity of diffuse hydrothermal flow by tracking moving refractive index anomalies. Geochem Geophys Geosyst. https://doi.org/10.1029/2010GC003227

Raffel M (2015) Background-oriented schlieren (BOS) techniques. Exp Fluids. https://doi.org/10.1007/s00348-015-1927-5

Settles GS (2018) Smartphone schlieren and shadowgraph imaging. Opt Laser Eng. https://doi.org/10.1016/j.optlaseng.2017.07.002

Settles GS, Hargather MJ (2017) A review of recent developments in schlieren and shadowgraph techniques. Meas Sci Technol. https ://doi.org/10.1088/1361-6501/aa5748

Smith NT, Heineck JT, Schairer ET (2017) Optical flow for flight and wind tunnel background oriented schlieren imaging. In: 55th AIAA aerospace sciences meeting, Grapevine, Texas. https://doi. org/10.2514/6.2017-0472

Publisher's Note Springer Nature remains neutral with regard to jurisdictional claims in published maps and institutional affiliations. 